\title{
General Smarr relation and first law of a NUT dyonic black hole
}

\author{
Zhaohui Chen ${ }^{\dagger}$ and Jie Jiang $\oplus^{*}$ \\ Department of Physics, Beijing Normal University, Beijing 100875, China
}

(Received 8 July 2019; published 8 November 2019)

\begin{abstract}
In this paper, we investigate the thermodynamics of the charged Newman-Unti-Tamburino black hole with the Misner strings present in the Einstein-Maxwell gravity. We show that the Misner charge $N$ can be obtained by performing Komar integration over the Misner strings in the presence of electric and magnetic charges and the corresponding Smarr relation and first law can also be naturally established based on this geometric perspective. Besides, we demonstrate that the electric and magnetic charges appearing in the first law and Smarr relation are contingent on the special choice of gauge freedom for the electric and magnetic potentials. We rederive two different versions of thermodynamics according to distinct choices of gauge and furthermore, we try to formulate more general thermodynamic laws under other arbitrary choices of gauge and deduce the conditions necessary to satisfy the Smarr relations and first laws simultaneously.
\end{abstract}

DOI: 10.1103/PhysRevD.100.104016

\section{INTRODUCTION}

As one of the most interesting solutions of general relativity, the Taub-NUT (Newman-Unti-Tamburino) spacetime $[1,2]$ has engendered lots of perplexing problems since its birth. In this spacetime exist two kinds of Killing vectors which, respectively, correspond to the Schwarzschild-like horizon and Misner string singularities on the north and south pole axes due to the existence of NUT parameter $n$. The Misner strings have also resulted in the appearance of spacetime regions with closed timelike curves in their vicinity.

To eschew these issues, Misner proposed that imposing the periodicity of time coordinates makes the string singularities unobservable [3]. Since then, the following studies on the Taub-NUT spacetime mostly focused on the Euclidean case. However, this manipulation also brings about many new problems. For instance, it leads to the existence of closed timelike curves everywhere and makes the spacetime geodesically incomplete [3-5] and moreover, when establishing thermodynamics of this spacetime, the NUT parameter $n$ is no longer a valid independent variable but rather a function of the Schwarzschild-like horizon radius $r_{h}$ due to the identification between the standard temperature formula and the reciprocal of the periodicity of

\footnotetext{
*Corresponding author. jiejiang@mail.bnu.edu.cn

chenzhaohui@mail.bnu.edu.cn

Published by the American Physical Society under the terms of the Creative Commons Attribution 4.0 International license. Further distribution of this work must maintain attribution to the author(s) and the published article's title, journal citation, and DOI. Funded by SCOAP ${ }^{3}$.
}

the Euclidean time coordinate [6]. This also results in the possibility that the thermodynamic volume is negative, and the entropy obtained is not in accord with the BekensteinHawking area law $[7,8]$.

However, recent studies have demonstrated that the removal of Misner strings by imposing the periodicity of the time coordinate in the Euclidean setup is unnecessary in fact. Clément et al. [9-11] illustrated that the Misner string singularities are far less problematic than previously thought and they argued that the Lorentzian Taub-NUT solution without imposing the Misner time periodicity condition is geodesically complete. At the same time, despite the existence of regions with closed timelike curves, causality is not violated at all for geodesic observers, implying that Lorentzian Taub-NUT spacetimes with the Misner strings present may be physical in essence.

Recently, D. Kubizňák et al. have formulated a reasonable thermodynamics of Lorentzian Taub-NUT-AdS spacetime with the Misner strings present [12]. They treated the NUT parameter $n$ as an independent variable, and thus introduced a new pair of conjugate thermodynamic quantities $\psi-N$, where $\psi$ is called the Misner potential and its conjugate term $N$ is named as the Misner gravitational charge. Such a new first law and Smarr relation just take the following forms:

$$
\begin{aligned}
\delta M & =T \delta S+\psi \delta N+V \delta P, \\
M & =2(T S-V P+\psi N) .
\end{aligned}
$$

Here, $M$ represents the mass of this spacetime, $T$ is the temperature of the Schwarzschild-like horizon, $S$ is the Bekenstein-Hawking entropy, $P$ is the cosmological constant pressure, and $V$ is the conjugate quantity of it. 
In this case, the cosmological constant is treated as a state parameter of the thermodynamic system. And this can be understood since the cosmological constant can be induced from a totally antisymmetric tensor field sourced by a membrane $[13,14]$. Originally, they just obtained the free energy by calculating the Euclidean action [15] to identify several thermodynamic terms and further determined the Misner charge $N$. Later, the geometrical interpretation on the two novel quantities $\psi$ and $N$ was assigned [16] and it was shown that the Misner potential is exactly the surface gravity of the Misner strings and the Misner charge just corresponds to the Komar integration over the tubes surrounding the Misner strings.

However, when extended to the charged Taub-NUT-AdS spacetime in the Einstein-Maxwell gravity [17-19], in this case the electric and magnetic charges, which were called "Nutty dyon," both exist and the magnitudes of them depend on the radius of the two-dimensional sphere [see (11) below]. Thus, an important problem occurs in the formulation of thermodynamics, namely, how to choose the proper electric charge $q_{e}$ and magnetic charge $q_{m}$ appearing in the first law and Smarr relation as thermodynamic quantities. Reference [20] gave two different types of thermodynamics of the Nutty dyonic black hole, and one is that the asymptotic electric charge and horizon magnetic charge are regarded as independent thermodynamic quantities, while as the other case, the horizon electric charge and the asymptotic magnetic charge are considered. Hence, two types of corresponding Smarr relations can be written as follows:

$$
\begin{aligned}
& M=2\left(T S-V P+\psi N^{(1)}\right)+\phi_{e} Q_{e}+\phi_{m} Q_{m}^{(+)}, \\
& M=2\left(T S-V P+\psi N^{(2)}\right)+\phi_{e} Q_{e}^{(+)}+\phi_{m} Q_{m} .
\end{aligned}
$$

Conspicuously, Misner charges for the charged case differ in these two distinct versions of thermodynamics. However, the expressions of Misner charges are still determined by means of calculating the Euclidean action for free energy [20] and the similar Komar integrations of them for Nutty dyonic black holes are not given in the previous literature. In addition, why one can formulate two different versions of thermodynamics in this spacetime and whether we can give an interpretation from geometrical notions deserve our deep exploration.

So, our purpose in this paper is to construct the thermodynamic laws of this Nutty dyonic black hole from the Komar integral perspective and recover the corresponding Misner charges given in [20]. Meanwhile, we illustrate that formulations of two different versions of thermodynamics are actually relevant to the gauge choices of electric and magnetic potentials and, moreover, we attempt to obtain more general types of thermodynamics based on arbitrary gauge choices from this perspective.
Our paper is organized as follows. In the next section, we briefly review the rudiments of the Taub-NUT-AdS spacetime and enumerate some basic thermodynamic quantities for the Nutty dyonic black hole. In Sec. III we present the geometrical philosophy to derive the Smarr relations and discuss how different gauge choices of electric and magnetic potentials influence the formulations of thermodynamics. Section IV is devoted to calculations of the Misner charges in two special distinct gauges. In Sec. V, we derive more general Smarr relations for the Nutty dyonic black hole under arbitrary choices of gauge and give essential conditions to satisfy the first law. Finally, conclusions are presented in Sec. VI.

\section{GEOMETRY OF CHARGED TAUB-NUT-ADS SPACETIME}

In this paper, we consider the charged Lorentzian TaubNUT-AdS solution of the four-dimensional EinsteinMaxwell gravity, where the action can be written as

$$
I=\frac{1}{16 \pi} \int_{M} d^{4} x \sqrt{-g}\left(R-2 \Lambda-F_{a b} F^{a b}\right),
$$

in which $\boldsymbol{F}=d \boldsymbol{A}$ is the electromagnetic strength and $R$ is the Ricci scalar of the spacetime. The equations of motion are given by

$$
\begin{aligned}
R_{a b}-\frac{1}{2} R g_{a b}+\Lambda g_{a b} & =T_{a b}, \\
d \boldsymbol{G} & =0,
\end{aligned}
$$

with the energy-momentum tensor of the Maxwell field

$$
T_{a b}=F_{a c} F_{b}^{c}+G_{a c} G_{b}^{c},
$$

and

$$
\boldsymbol{G}=\star \boldsymbol{F} .
$$

The electric and magnetic charges inside a two-dimensional surface $S^{2}$ can be defined as

$$
q_{e}\left[S^{2}\right]=\frac{1}{4 \pi} \int_{S^{2}} \boldsymbol{G}, \quad q_{m}\left[S^{2}\right]=\frac{1}{4 \pi} \int_{S^{2}} \boldsymbol{F} .
$$

According to the equations of motion (5), the Taub-NUTAdS solution can be read off $[8,19]$

$$
\begin{aligned}
d s^{2}= & -f(r)(d t+2 n \cos \theta d \phi)^{2}+\frac{d r^{2}}{f(r)} \\
& +\left(r^{2}+n^{2}\right)\left(d \theta^{2}+\sin ^{2} \theta d \phi^{2}\right) \\
\boldsymbol{A}= & -\left[h(r)-h_{0}\right] d t+2 h(r) n \cos \theta d \phi
\end{aligned}
$$

where 


$$
f(r)=\frac{r^{2}-2 m r-n^{2}+4 n^{2} g^{2}+e^{2}}{r^{2}+n^{2}}-\frac{3 n^{4}-6 n^{2} r^{2}-r^{4}}{l^{2}\left(r^{2}+n^{2}\right)}
$$

is the blackening factor,

$$
h(r)=\frac{e r}{r^{2}+n^{2}}+\frac{g\left(r^{2}-n^{2}\right)}{r^{2}+n^{2}},
$$

and $h_{0}$ is some arbitrary constant, which reflects the gauge freedom of the electromagnetic field. Besides, $n$ and $m$ are the NUT and mass parameters, $e$ and $g$ are the electric and magnetic parameters, and $l$ is the anti-de Sitter (AdS) radius with $\Lambda=-3 / l^{2}$.

Performing the integration over a sphere of radius $r$ according to Eq. (8), we can obtain the electric and magnetic charges inside this sphere

$$
\begin{aligned}
q_{e}(r) & =\frac{e\left(r^{2}-n^{2}\right)-4 g r n^{2}}{r^{2}+n^{2}}, \\
q_{m}(r) & =\frac{2 n\left[e r+g\left(r^{2}-n^{2}\right)\right]}{r^{2}+n^{2}} .
\end{aligned}
$$

One can note that disparate with the common results of the dyonic solutions, the charges vary with the radius of the sphere $S^{2}$, which can be easily understood since the sphere $S^{2}$ is not a close surface due to the existence of the Misner singularities. And two kinds of special values of these charges are, respectively, the asymptotic $(r \rightarrow \infty)$ charges:

$Q_{e}=q_{e}(r \rightarrow \infty)=e, \quad Q_{m}=q_{m}(r \rightarrow \infty)=2 g n$,

and the horizon $\left(r=r_{h}\right)$ charges:

$$
Q_{e}^{(+)}=q_{e}\left(r_{h}\right), \quad Q_{m}^{(+)}=q_{m}\left(r_{h}\right),
$$

where $r_{h}$ is the largest root of $f\left(r_{h}\right)=0$. By using the conformal method [21], the mass of this black hole is obtained as

$$
M=m,
$$

while the total angular momentum of the spacetime vanishes. According to the line element (9), the Killing vector of the horizon is manifestly given by

$$
k^{a}=\left(\frac{\partial}{\partial t}\right)^{a}
$$

And the temperature of this Schwarzschild-like Killing horizon is given by the surface gravity

$$
\begin{aligned}
T & =\frac{\kappa_{h}}{2 \pi}=\frac{f^{\prime}\left(r_{h}\right)}{4 \pi} \\
& =\frac{1}{4 \pi r_{h}}\left(1+\frac{3\left(r_{h}^{2}+n^{2}\right)}{l^{2}}-\frac{e^{2}+4 n^{2} g^{2}}{r_{h}^{2}+n^{2}}\right) .
\end{aligned}
$$

The entropy can be identified with the horizon area law:

$$
S=\frac{A}{4}=\pi\left(r_{h}^{2}+n^{2}\right) .
$$

Moreover, there are yet other Killing horizons present in the spacetime, which are separately located on the north and south pole axes with the Killing vectors

$$
\xi_{ \pm}^{a}=k^{a} \mp \frac{1}{2 n} \varphi^{a}
$$

with $\varphi^{a}=(\partial / \partial \phi)^{a}$. The corresponding surface gravity can be calculated by the standard formula

$$
\kappa_{\xi}^{2}=\frac{1}{2} \nabla_{a} \xi_{b} \nabla^{a} \xi^{b},
$$

which gives the Misner potentials

$$
\psi=\psi_{ \pm}=\frac{\kappa_{\xi}}{2 \pi}=\frac{1}{8 \pi n} .
$$

In what follows, we will identify the conjugate pairs appearing in the Smarr relations with the Komar integrations and give different types of electric and magnetic terms from the geometric perspective.

\section{SMARR RELATION WITH ELECTRIC AND MAGNETIC CHARGES}

In [20], the authors gave the consistent first law and Smarr relation of the Nutty dyonic black hole containing the electric and magnetic charges based on the thermodynamic consideration. Inspired by the uncharged case [16] and the Taub-NUT solution with rotation [22], in this section we would like to derive a general Smarr relation and then apply it to reexpress the thermodynamic quantities obtained in [20] from the Komar integral perspective. The key point to achieve it is the decomposition of the mass [23]. Now, for the Killing vector $k^{a}$, we have

$$
d(\star k)=0,
$$

which implies that there locally exists a 2 -form $\omega$, such that

$$
\star k=d \omega .
$$

Then, together with the equation of motion (5), utilizing the facts that $k^{a}$ is a Killing vector and the sum of cyclic permutations of the last three indices in $R_{b c d}^{a}$ vanishes, we thus have the following identity: 


$$
\nabla_{a} \nabla^{a} k^{b}=-R^{b}{ }_{a} k^{a}=-\Lambda k^{b}-T^{a b} k_{a} .
$$

We observe that for the charged case, the energymomentum term will be devoted to constructing the electric and magnetic charge terms in the Smarr relation. Thus, we need to transform this term into differential forms using the expression (6) of $T^{a b}$ and then give the Komar integrals of the electric and magnetic terms. Moreover, from the equation of motion $d \boldsymbol{G}=0$ of the electromagnetic field, we can see that the on-shell value of $\boldsymbol{G}$ is a closed 2-form, which implies that there exists a 1-form $\boldsymbol{B}$ such that $\boldsymbol{G}=d \boldsymbol{B}$. We can note that the newly defined vector potential $\boldsymbol{B}$ also allows a gauge freedom. So, substituting Eq. (6) into the above expression, the second term becomes

$$
\begin{aligned}
T^{a b} k_{a} & =k_{a} F^{a}{ }_{c} F^{b c}+k_{a} G^{a}{ }_{c} G^{b c} \\
& =F^{b c} k_{a} \nabla^{a} A_{c}-F^{b c} k_{a} \nabla_{c} A^{a}+G^{b c} k_{a} \nabla^{a} B_{c}-G^{b c} k_{a} \nabla_{c} B^{a} \\
& =-F^{b c} A_{a} \nabla_{c} k^{a}-F^{b c} k^{a} \nabla_{c} A_{a}-G^{b c} B_{a} \nabla_{c} k^{a}-G^{b c} k^{a} \nabla_{c} B_{a} \\
& =-F^{b c} \nabla_{c} \Phi_{e}-G^{b c} \nabla_{c} \Phi_{m} \\
& =\nabla_{a}\left(F^{a b} \Phi_{e}+G^{a b} \Phi_{m}\right),
\end{aligned}
$$

where we have used the fact that Lie derivatives of vector potentials $\boldsymbol{A}$ and $\boldsymbol{B}$ along the vector $k^{a}$ vanish, namely, $\mathcal{L}_{k} A=0$ and $\mathcal{L}_{k} B=0$, and denote the contractions of the Killing vector $k^{a}$ with two vector potentials as

$$
\Phi_{e}=k^{a} A_{a}, \quad \Phi_{m}=k^{a} B_{a},
$$

which can be regarded as the electric and magnetic potentials of the Maxwell field. And the counterparts of the black hole can be defined as

$\phi_{e}=\Phi_{e}(\infty)-\Phi_{e}\left(r_{h}\right), \quad \phi_{m}=\Phi_{m}(\infty)-\Phi_{m}\left(r_{h}\right)$.

Then, Eq. (23) together with (24) yields

$$
\nabla_{a}\left(\nabla^{a} k^{b}+F^{a b} \Phi_{e}+G^{a b} \Phi_{m}\right)=-\Lambda k^{b} .
$$

Using the language of differential forms, we have

$$
d\left(\star d \boldsymbol{k}+2 \Phi_{e} \boldsymbol{G}-2 \Phi_{m} \boldsymbol{F}\right)+2 \Lambda \star \boldsymbol{k}=0,
$$

which implies

$$
d\left(\star d \boldsymbol{k}+2 \Phi_{e} \boldsymbol{G}-2 \Phi_{m} \boldsymbol{F}+2 \Lambda \boldsymbol{\omega}\right)=0 .
$$

Integrating over a three-dimensional hypersurface $\Sigma$ and using the Stokes theorem, it can be expressed by some boundary quantities,

$$
\begin{aligned}
0 & =\int_{\Sigma} d\left(\star d \boldsymbol{k}+2 \Phi_{e} \boldsymbol{G}-2 \Phi_{m} \boldsymbol{F}+2 \Lambda \boldsymbol{\omega}\right) \\
& =\int_{\partial \Sigma}\left(\star d \boldsymbol{k}+2 \Phi_{e} \boldsymbol{G}-2 \Phi_{m} \boldsymbol{F}+2 \Lambda \boldsymbol{\omega}\right) .
\end{aligned}
$$

For the normal black hole, the hypersurface $\Sigma$ is bounded by the spheres $S_{h}$ on the horizon and $S_{\infty}$ at asymptotic infinity. However, when the Misner strings are present, there exist the Misner string singularities which are located at $\theta=0$ and $\theta=\pi$. Then, the decomposition will depend on the Misner strings. Therefore, we also need introduce two Misner string tubes $T_{+}$and $T_{-}$which are located at $\theta=\varepsilon$ and $\theta=\pi-\varepsilon$ (see Fig. 1) with infinitesimal parameter $\varepsilon$, respectively. After taking into account the orientation of the boundaries, we have

$$
\partial \Sigma=T_{+}+S_{\infty}-T_{-}-S_{h}
$$

Thus, the integral (30) can be decomposed into the following integrations over several pieces of boundaries:

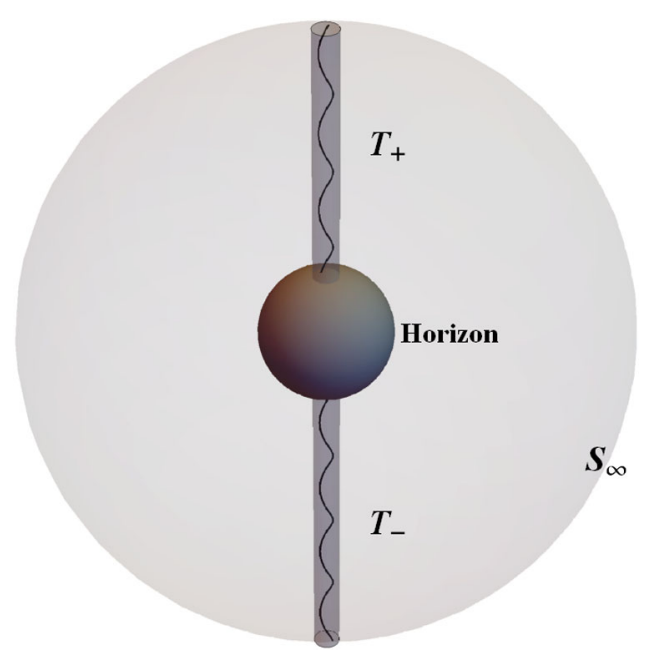

FIG. 1. Taub-NUT-AdS boundaries: Misner tubes [16], as a result of the effect of NUT parameter $n$, besides the standard boundaries on the Schwarzschild-like horizon $S_{h}$ and at infinity $S_{\infty}$, the two Misner tubes $T_{ \pm}$of radius $\varepsilon$ surrounding the symmetric strings are located at the north and south pole axes, $\cos \theta= \pm 1$. 


$$
\begin{aligned}
0= & \int_{S_{\infty}}(\star d \boldsymbol{k}+2 \Lambda \boldsymbol{\omega})-\int_{S_{h}} \star d \boldsymbol{k} \\
& +2 \int_{S_{\infty}}\left(\Phi_{e} \boldsymbol{G}-\Phi_{m} \boldsymbol{F}\right)-2 \int_{S_{h}}\left(\Phi_{e} \boldsymbol{G}-\Phi_{m} \boldsymbol{F}\right) \\
& -2 \Lambda\left(\int_{S_{h}} \boldsymbol{\omega}+\Omega_{+}^{\left(r=r_{h}\right)}-\Omega_{-}^{\left(r=r_{h}\right)}\right) \\
& +\int_{T_{+}}\left(\star d \boldsymbol{k}+2 \Lambda \Omega_{+}^{(r=\infty)}+2 \Phi_{e} \boldsymbol{G}-2 \Phi_{m} \boldsymbol{F}\right) \\
& -\int_{T_{-}}\left(\star d \boldsymbol{k}+2 \Lambda \Omega_{+}^{(r=\infty)}+2 \Phi_{e} \boldsymbol{G}-2 \Phi_{m} \boldsymbol{F}\right) .
\end{aligned}
$$

In the same way as the uncharged case [16], we also denote

$$
\int_{T_{ \pm}} \boldsymbol{\omega}=\Omega_{ \pm}^{(r=\infty)}-\Omega_{ \pm}^{\left(r=r_{h}\right)},
$$

splitting the integral into the $r \rightarrow \infty$ and $r=r_{h}$ parts. From Eq. (32) we can see that the existence of the electromagnetic field provides a modification for the Misner charge apart from furnishing the electric and magnetic terms. By identifying the thermodynamic mass $M$, cosmological constant pressure $P$, and the product of entropy and temperature with corresponding Komar integrals [23],

$$
\begin{aligned}
M & =-\frac{1}{8 \pi} \int_{S_{\infty}}(\star d \boldsymbol{k}+2 \Lambda \omega), \quad P=-\frac{\Lambda}{8 \pi}, \\
T S & =-\frac{1}{16 \pi} \int_{H} \star d \boldsymbol{k},
\end{aligned}
$$

Eq. (32) can be simplified as

$$
M=2\left(T S-P V+\psi N^{\Phi}\right)+\left[\Phi_{e} q_{e}-\Phi_{m} q_{m}\right]_{r_{h}}^{\infty} .
$$

With the similar consideration as [16], we also define the thermodynamic volume and Misner gravitational charge as

$$
\begin{aligned}
V & =-\left(\int_{S_{h}} \boldsymbol{\omega}+\Omega_{+}^{\left(r=r_{h}\right)}-\Omega_{-}^{\left(r=r_{h}\right)}\right), \\
N^{\Phi} & =N_{+}^{\Phi}+N_{-}^{\Phi}
\end{aligned}
$$

with

$N_{ \pm}^{\Phi}= \pm \frac{1}{16 \pi \psi} \int_{T_{ \pm}}\left(\star d \boldsymbol{k}+2 \Lambda \Omega_{ \pm}^{(r=\infty)}+2 \Phi_{e} \boldsymbol{G}-2 \Phi_{m} \boldsymbol{F}\right)$.

So far, we have derived a more general Smarr relation for the Nutty dyonic black hole. Normally, for some black holes with charges $q_{e}$ and $q_{m}$ being constants, we know that the gauge freedoms of $\Phi_{e}$ and $\Phi_{m}$ are trivial from Eq. (26). For this case, since $q_{e}$ and $q_{m}$ are no longer constants but rather functions of the radius $r$ of sphere $S^{2}$ as demonstrated in Eq. (11), different gauge choices of the electric and magnetic potentials $\Phi_{e}$ and $\Phi_{m}$ will give distinct electric and magnetic terms appearing in the Smarr relations and as is shown in Eq. (37), the corresponding Misner charges $N^{\Phi}$ will also make variations accordingly. Thus, we realize that in order to obtain the similar decompositions as the Smarr relations (2) and (3), we need to choose some special gauges. If choosing the gauge $\Phi_{e}=\Phi_{e}^{(1)}, \Phi_{m}=\Phi_{m}^{(1)}$ such that

$$
\Phi_{e}^{(1)}\left(r_{h}\right)=0, \quad \Phi_{m}^{(1)}(\infty)=0,
$$

we have $\Phi_{e}^{(1)}(\infty)=\phi_{e}, \Phi_{m}^{(1)}\left(r_{h}\right)=-\phi_{m}$, and this choice ensures that asymptotic electric charge $Q_{e}$ and horizon magnetic charge $Q_{m}^{(+)}$appear in the first law and Smarr relation. Then Eq. (35) becomes

$$
M=2\left(T S-P V+\psi N^{(1)}\right)+\phi_{e} Q_{e}+\phi_{m} Q_{m}^{(+)},
$$

where $N^{(1)}=N^{\Phi^{(1)}}$ is the value of the Misner charge under the above gauge (38). Equation (39) gives the same decomposition as the Smarr relation (2) by the Komar integral.

Based on another choice of gauge, we can formulate an alternative Smarr relation (3), where the electric charge is taken on the horizon and the magnetic counterpart is taken at infinity. Here, on the contrary to Eq. (38), we should choose the gauge $\Phi_{e}=\Phi_{e}^{(2)}, \Phi_{m}=\Phi_{m}^{(2)}$ such that

$$
\Phi_{m}^{(2)}\left(r_{h}\right)=0, \quad \Phi_{e}^{(2)}(\infty)=0 .
$$

Thus, we have $\Phi_{e}^{(2)}\left(r_{h}\right)=-\phi_{e}, \Phi_{m}^{(2)}(\infty)=\phi_{m}$ and the decomposition becomes

$$
M=2\left(T S-P V+\psi N^{(2)}\right)+\phi_{e} Q_{e}^{(+)}+\phi_{m} Q_{m},
$$

which is exactly the Smarr relation (3). And here, $N^{(2)}$ is the value of $N^{\Phi}$ under the gauge (40). The above discussion shows that the corresponding Misner charges obtained in [20] can be expressed as some Komar integrations (37) in two special choices of gauge (38) and (40).

Finally, in order to ensure the rationality of these quantities, we should check the validity of the first law of thermodynamics, i.e.,

$$
\begin{aligned}
& \delta M=T \delta S+V \delta P+\psi \delta N^{(1)}+\phi_{e} \delta Q_{e}+\phi_{m} \delta Q_{m}^{(+)}, \\
& \delta M=T \delta S+V \delta P+\psi \delta N^{(2)}+\phi_{e} \delta Q_{e}^{(+)}+\phi_{m} \delta Q_{m} .
\end{aligned}
$$




\section{FIRST LAW WITH VARIABLE MISNER CHARGES UNDER SOME SPECIAL GAUGE CHOICES}

In the former section, we elaborate on the basic strategy for establishing the thermodynamics of the Nutty dyonic black hole from the geometric perspective and show that different gauge choices of $\Phi_{e}$ and $\Phi_{m}$ will give distinct types of Smarr relations. From Eq. (37), we know that Misner charges are also subject to influences from the gauge freedoms as well as the electric and magnetic terms. And below, we are devoted to calculating the Komar integrations of Misner charges and other thermodynamic quantities to recover the results in Ref. [20] in two special gauges. Besides, the corresponding first law (42) will be examined. According to Eq. (9), we immediately obtain the electromagnetic field tensor

$$
\begin{aligned}
\boldsymbol{F}= & -\frac{e\left(n^{2}-r^{2}\right)+4 n^{2} g r}{\left(r^{2}+n^{2}\right)^{2}} d r \wedge d t+\frac{2 n\left[e r+g\left(r^{2}-n^{2}\right)\right]}{r^{2}+n^{2}} \\
& \times \sin \theta d \theta \wedge d \phi-2 n \frac{e\left(n^{2}-r^{2}\right)+4 n^{2} g r}{\left(r^{2}+n^{2}\right)^{2}} \cos \theta d r \wedge d \phi .
\end{aligned}
$$

Then, we can instantly obtain

$$
\begin{aligned}
\boldsymbol{G}= & \frac{2 n\left[g\left(n^{2}-r^{2}\right)-e r\right]}{\left(r^{2}+n^{2}\right)^{2}} d r \wedge d t+\frac{e\left(r^{2}-n^{2}\right)-4 n^{2} g r}{r^{2}+n^{2}} \\
& \times \sin \theta d \theta \wedge d \phi+\frac{4 n^{2}\left[g\left(n^{2}-r^{2}\right)-e r\right]}{\left(r^{2}+n^{2}\right)^{2}} \cos \theta d r \wedge d \phi .
\end{aligned}
$$

From this, it is not difficult to verify the newly defined vector potential

$$
\boldsymbol{B}=\left[p(r)-p_{0}\right] d t+2 n p(r) \cos \theta d \phi
$$

with

$$
p(r)=\frac{4 n^{2} g r-e\left(r^{2}-n^{2}\right)}{2 n\left(r^{2}+n^{2}\right)},
$$

and arbitrary constant $p_{0}$, which should be determined when we fix the gauge. At the same time, we evaluate the Hodge dual of $k^{a}$, namely,

$$
\begin{aligned}
\star d \boldsymbol{k}= & -\frac{2 f n}{r^{2}+n^{2}} d r \wedge(d t+2 n \cos \theta d \phi) \\
& -\sin \theta\left(r^{2}+n^{2}\right) f^{\prime} d \theta \wedge d \phi .
\end{aligned}
$$

Then from Eq. (22), we note that $\omega$ cannot be specified uniquely and to ensure that the integration (34) gives the genuine mass $m$, one can employ the method of the KillingYano tensor [16] (details see also [24,25]) to obtain

$$
\begin{array}{r}
\omega=-\frac{n}{3} d r \wedge(d t+2 n \cos \theta d \phi) \\
-\frac{r}{3} \sin \theta\left(r^{2}+n^{2}\right) d \theta \wedge d \phi .
\end{array}
$$

From integration (33), we also notice that $\Omega_{ \pm}^{r=\infty}$ and $\Omega_{ \pm}^{r=r_{h}}$ are determined freely by a constant. Likewise, here we also choose $\Omega_{ \pm}^{r=\infty}$ only containing the divergent term. By integrating on (46), we have

$$
\Omega_{ \pm}^{r=\infty}=\mp \lim _{r \rightarrow \infty} \frac{4 \pi n^{2}}{3} r, \quad \Omega_{ \pm}^{r=r_{h}}=\mp \frac{4 \pi n^{2}}{3} r_{h} .
$$

Using the above results, we can further obtain the thermodynamic volume

$$
V=\frac{4 \pi r_{h}^{3}}{3}\left(1+\frac{3 n^{2}}{r_{h}^{2}}\right)
$$

which is in accord with the uncharged case [16]. Next, Eq. (25) gives electric and magnetic potential by

$$
\Phi_{e}=h_{0}-h(r), \quad \Phi_{m}=p(r)-p_{0} .
$$

First, we consider the gauge (38). Under this gauge, we have

$\Phi_{e}^{(1)}=\frac{2 g n^{2}-e r}{r^{2}+n^{2}}-\frac{2 g n^{2}-e r_{h}}{r_{h}^{2}+n^{2}}, \quad \Phi_{m}^{(1)}=\frac{n(2 g r+e)}{r^{2}+n^{2}}$.

Hence, the electric and magnetic potentials of the black hole can be written as

$$
\phi_{e}=\frac{e r_{h}-2 g n^{2}}{r_{h}^{2}+n^{2}}, \quad \phi_{m}=\frac{n\left(2 g r_{h}+e\right)}{r_{h}^{2}+n^{2}}
$$

After determining the electric charge $Q_{e}$ and magnetic counterpart $Q_{m}^{(+)}$, we begin to tackle the Misner terms and obtain that

$$
\begin{aligned}
\int_{T_{+}} \star d \boldsymbol{k}= & -\left.\frac{8 \pi n^{2} r}{l^{2}}\right|_{r \rightarrow \infty}+\frac{8 \pi n^{2} r_{h}}{l^{2}}-\frac{2 \pi^{2}\left(e^{2}+4 g^{2} n^{2}\right)}{n} \\
& +\frac{4 \pi\left[2 m n^{2}+\left(e^{2}+4 g^{2} n^{2}-2 n^{2}\right) r_{h}\right]}{r_{h}^{2}+n^{2}}-\frac{32 \pi n^{4} r_{h}}{l^{2}\left(r_{h}^{2}+n^{2}\right)} \\
& +\frac{4 \pi\left(e^{2}+4 g^{2} n^{2}\right) \arctan \left(\frac{r_{h}}{n}\right)}{n} .
\end{aligned}
$$

From this result, we find that $2 \Lambda \Omega_{+}^{r=\infty}=\left.\left(8 \pi n^{2} r / l^{2}\right)\right|_{r \rightarrow \infty}$, exactly canceling out the divergence term. And then, we perform the integrals on field strength tensors over the Misner string under the choice of gauge (38) and obtain the following results: 


$$
\begin{aligned}
\int_{T_{+}} 2 \Phi_{e}^{(1)} \boldsymbol{G}= & \frac{\pi^{2}\left(e^{2}+4 g^{2} n^{2}\right)}{n}-\frac{2 \pi r_{h}\left(e^{2}-4 g^{2} n^{2}\right)\left(r_{h}^{2}-n^{2}\right)}{\left(r_{h}^{2}+n^{2}\right)^{2}} \\
& +\frac{16 \pi e g n^{2} r_{h}^{2}}{\left(r_{h}^{2}+n^{2}\right)^{2}}-\frac{2 \pi\left(e^{2}+4 g^{2} n^{2}\right) \arctan \left(\frac{r_{h}}{n}\right)}{n} \\
& -\frac{8 n^{2} \pi\left[e r_{h}+g\left(r_{h}^{2}-n^{2}\right)\right]\left(e+2 g r_{h}\right)}{\left(r_{h}^{2}+n^{2}\right)^{2}}, \\
\int_{T_{+}} 2 \Phi_{m}^{(1)} \boldsymbol{F}= & -\frac{\pi^{2}\left(e^{2}+4 g^{2} n^{2}\right)}{n}+\frac{8 \pi g n^{2}\left(e+g r_{h}\right)\left(r_{h}^{2}-n^{2}\right)}{\left(r_{h}^{2}+n^{2}\right)^{2}} \\
& +\frac{2 \pi e^{2} r_{h}\left(r_{h}^{2}+3 n^{2}\right)}{\left(r_{h}^{2}+n^{2}\right)^{2}} \\
& +\frac{2 \pi\left(e^{2}+4 g^{2} n^{2}\right) \arctan \left(\frac{r_{h}}{n}\right)}{n} .
\end{aligned}
$$

Hence, according to Eq. (37), the above calculations yield

$$
\begin{aligned}
16 \pi \psi N_{+}^{(1)} & =\int_{T_{+}}\left(\star d \boldsymbol{k}+\frac{4 \Lambda}{n-2} \Omega_{+}^{(r=\infty)}+2 \Phi_{e}^{(1)} \boldsymbol{G}-2 \Phi_{m}^{(1)} \boldsymbol{F}\right) \\
= & -\frac{4 n^{2} \pi}{r_{h}}\left[1+\frac{3\left(n^{2}-r_{h}^{2}\right)}{l^{2}}+\frac{\left(r_{h}^{2}-n^{2}\right)\left(e^{2}+4 e g r_{h}\right)}{\left(r_{h}^{2}+n^{2}\right)^{2}}\right. \\
& \left.-\frac{4 n^{2} g^{2}\left(3 r_{h}^{2}+n^{2}\right)}{\left(r_{h}^{2}+n^{2}\right)^{2}}\right]
\end{aligned}
$$

By considering $N_{+}^{(1)}=N_{-}^{(1)}$ and using $\psi$ in Eq. (20), the Misner charge can be ultimately obtained as

$$
\begin{aligned}
N^{(1)}= & -\frac{4 n^{3} \pi}{r_{h}}\left[1+\frac{3\left(n^{2}-r_{h}^{2}\right)}{l^{2}}+\frac{\left(r_{h}^{2}-n^{2}\right)\left(e^{2}+4 e g r_{h}\right)}{\left(r_{h}^{2}+n^{2}\right)^{2}}\right. \\
& \left.-\frac{4 n^{2} g^{2}\left(3 r_{h}^{2}+n^{2}\right)}{\left(r_{h}^{2}+n^{2}\right)^{2}}\right] .
\end{aligned}
$$

It is now easy to verify that with these quantities the first law is satisfied,

$$
\delta M=T \delta S+\phi_{e} \delta Q_{e}+\phi_{m} \delta Q_{m}^{(+)}+\psi \delta N^{(1)}+V \delta P .
$$

Analogously, under the gauge (40), the electric and magnetic potentials can be written as

$$
\begin{aligned}
& \Phi_{e}^{(2)}(r)=\frac{2 g n^{2}-e r}{r^{2}+n^{2}}, \\
& \Phi_{m}^{(2)}(r)=\frac{n(2 g r+e)}{r^{2}+n^{2}}-\frac{n\left(2 g r_{h}+e\right)}{r_{h}^{2}+n^{2}} .
\end{aligned}
$$

Through similar calculations, we obtain that

$$
\begin{aligned}
\int_{T_{+}} 2 \Phi_{e}^{(2)} \boldsymbol{G}= & \frac{\pi^{2}\left(e^{2}+4 g^{2} n^{2}\right)}{n}+\frac{2 \pi\left(4 e g n^{2}-e^{2} r_{h}\right)\left(r_{h}^{2}-n^{2}\right)}{\left(r_{h}^{2}+n^{2}\right)^{2}} \\
& -\frac{8 \pi g^{2} n^{2} r_{h}\left(r_{h}^{2}+3 n^{2}\right)}{\left(r_{h}^{2}+n^{2}\right)^{2}} \\
& -\frac{2 \pi\left(e^{2}+4 g^{2} n^{2}\right) \arctan \left(\frac{r_{h}}{n}\right)}{n}, \\
\int_{T_{+}} 2 \Phi_{m}^{(2)} \boldsymbol{F}= & -\frac{\pi^{2}\left(e^{2}+4 g^{2} n^{2}\right)}{n}+\frac{2 \pi r_{h}\left(4 g^{2} n^{2}-e^{2}\right)\left(r_{h}^{2}-n^{2}\right)}{\left(r_{h}^{2}+n^{2}\right)^{2}} \\
& +\frac{16 \pi e g n^{2} r_{+}^{2}}{\left(r_{h}^{2}+n^{2}\right)^{2}}+\frac{2 \pi\left(e^{2}+4 g^{2} n^{2}\right) \arctan \left(\frac{r_{h}}{n}\right)}{n} \\
& +\frac{4 \pi\left[4 n^{2} g r_{h}-e\left(r_{h}^{2}-n^{2}\right)\right]\left(-2 g n^{2}+e r_{h}\right)}{\left(r_{h}^{2}+n^{2}\right)^{2}} .
\end{aligned}
$$

Again, according to the Komar integral of the Misner charge (37), we can obtain that

$$
\begin{aligned}
N^{(2)}= & -\frac{4 n^{3} \pi}{r_{h}}\left[1+\frac{3\left(n^{2}-r_{h}^{2}\right)}{l^{2}}+\frac{\left(r_{h}^{2}-n^{2}\right)\left(4 n^{2} g^{2}-4 e g r_{h}\right)}{\left(r_{h}^{2}+n^{2}\right)^{2}}\right. \\
& \left.-\frac{e^{2}\left(3 r_{h}^{2}+n^{2}\right)}{\left(r_{h}^{2}+n^{2}\right)^{2}}\right]
\end{aligned}
$$

And the corresponding first law is also satisfied:

$\delta M=T \delta S+\phi_{e} \delta Q_{e}^{(+)}+\phi_{m} \delta Q_{m}+\psi \delta N^{(2)}+V \delta P$.

The above two procedures are performed based on two different choices of gauge from this geometric perspective. One case is that the electric potential on the horizon and magnetic potential at infinity vanish so that the asymptotic electric charge and horizon magnetic charge are considered as variables of thermodynamics, and the other is just a contrary gauge to regard the asymptotic magnetic charge and horizon electric charge as the independent thermodynamic quantities. Under these two gauges, different Misner charges $N^{(1)}$ and $N^{(2)}$ are obtained by Komar integrations.

\section{GENERAL THERMODYNAMIC LAWS WITH MIXED INFINITY/HORIZON CHARGES UNDER OTHER GAUGE CHOICES}

The analyses in Secs. III and IV naturally raise an interesting question: could we formulate a more general full cohomogeneity first law and Smarr relation, in which case the electric charge is the mixture of corresponding charges at asymptotic infinity and on the horizon, and so is the magnetic charge? We find that it is feasible for the general Smarr relation (35), since we can always make proper decompositions for the $\Phi_{e}$ and $\Phi_{m}$ in arbitrary gauges. However, the consistent first laws will only hold under certain conditions. Next, we first construct the general Smarr relation in forms of mixed infinity/horizon charges, 
and it is not hard to see that the electric potential in any gauge can be decomposed as follows:

$$
\begin{aligned}
\Phi_{e} & =\Phi_{e}^{(1)}-\alpha \phi_{e} \\
& =\Phi_{e}^{(1)}+\alpha\left(\Phi_{e}^{(2)}-\Phi_{e}^{(1)}\right) \\
& =(1-\alpha) \Phi_{e}^{(1)}+\alpha \Phi_{e}^{(2)} .
\end{aligned}
$$

Similarly for the magnetic potential, we can also choose the gauge as

$$
\Phi_{m}=(1-\beta) \Phi_{m}^{(1)}+\beta \Phi_{m}^{(2)} .
$$

Here $\alpha$ and $\beta$ could be either constants or functions of independent variables $\left\{e, g, r_{h}, n, l\right\}$. Thus, the decomposition (35) can be written as

$$
M=2\left(T S-P V+\psi N^{\Phi}\right)+\phi_{e} \tilde{Q}_{e}+\phi_{m} \tilde{Q}_{m},
$$

where the mixed electric and magnetic charge are defined by

$$
\begin{gathered}
\tilde{Q}_{e}=\alpha Q_{e}^{(+)}+(1-\alpha) Q_{e}, \\
\tilde{Q}_{m}=(1-\beta) Q_{m}^{(+)}+\beta Q_{m} .
\end{gathered}
$$

In this case, we can also expressed the novel Misner charge as the mixture of $N^{(1)}$ and $N^{(2)}$.

$$
N^{\Phi}=\gamma N^{(1)}+(1-\gamma) N^{(2)},
$$

where $\gamma$ is a constant which should be determined by demanding the Smarr relation (61). To satisfy this relation, we can deduce that

$$
\begin{aligned}
& \int_{T_{+}}\left[\alpha \Phi_{e}^{(2)} \boldsymbol{G}+(1-\alpha) \Phi_{e}^{(1)} \boldsymbol{G}\right]-\left[(1-\beta) \Phi_{m}^{(1)} \boldsymbol{F}+\beta \Phi_{m}^{(2)} \boldsymbol{F}\right] \\
& =\gamma \int_{T_{+}}\left(\Phi_{e}^{(1)} \boldsymbol{G}-\Phi_{m}^{(1)} \boldsymbol{F}\right)+(1-\gamma) \int_{T_{+}}\left(\Phi_{e}^{(2)} \boldsymbol{G}-\Phi_{m}^{(2)} \boldsymbol{F}\right) .
\end{aligned}
$$

A further calculation shows that

$$
\begin{aligned}
& (\alpha+\gamma-1) \int_{T_{+}}\left(\Phi_{e}^{(2)}-\Phi_{e}^{(1)}\right) \boldsymbol{G} \\
& \quad=(\beta+\gamma-1) \int_{T_{+}}\left(\Phi_{m}^{(2)}-\Phi_{m}^{(1)}\right) \boldsymbol{F} .
\end{aligned}
$$

After performing integrals of two sides, we find that

$$
\begin{aligned}
\int_{T_{+}}\left(\Phi_{e}^{(2)}-\Phi_{e}^{(1)}\right) \boldsymbol{G} & =-\int_{T_{+}}\left(\Phi_{m}^{(2)}-\Phi_{m}^{(1)}\right) \boldsymbol{F} \\
& =-\frac{4 \pi n^{2}\left(2 g n^{2}-e r_{h}\right)\left(2 g r_{h}+e\right)}{\left(r_{h}^{2}+n^{2}\right)^{2}} .
\end{aligned}
$$

Hence, we obtain that

$$
\gamma=1-\frac{\alpha+\beta}{2} .
$$

At present, we have accomplished the formulation of the general Smarr relation after determining the value of $\gamma$. However, these variables have to satisfy the first law of thermodynamics, namely,

$$
\delta M=T \delta S+\phi_{e} \delta \tilde{Q}_{e}+\phi_{m} \delta \tilde{Q}_{m}+\psi \delta \tilde{N}+V \delta P .
$$

We can reexpress the first law in terms of independent physical parameters $\left\{r_{h}, e, g, l, n\right\}$. For example, the lefthand side becomes

$$
\delta M=\frac{\partial M}{\partial r_{h}} \delta r_{h}+\frac{\partial M}{\partial e} \delta e+\frac{\partial M}{\partial g} \delta g+\frac{\partial M}{\partial n} \delta n+\frac{\partial M}{\partial l} \delta l .
$$

Analogous manipulations are made for the right-hand side and therefore, Eq. (66) is equivalent to the following equations:

$$
\begin{aligned}
& \frac{n^{2}}{\left(r_{h}^{2}+n^{2}\right)^{2}}\left\{\left[2 e g\left(n^{2}-r_{h}^{2}\right)+\left(4 g^{2} n^{2}-e^{2}\right) r_{h}\right] \frac{\partial}{\partial r_{h}}(\alpha+\beta)\right. \\
& \left.+\left(e^{2}+4 g^{2} n^{2}\right)(\alpha-\beta)\right\}=0, \\
& \frac{n^{2}}{\left(r_{h}^{2}+n^{2}\right)^{2}}\left\{\left[\left(4 g^{2} n^{2}-e^{2}\right) r_{h}+2 e g\left(n^{2}-r_{h}^{2}\right)\right] \frac{\partial}{\partial e}(\alpha+\beta)\right. \\
& \left.\quad+2 g\left(r_{h}^{2}+n^{2}\right)(\alpha-\beta)\right\}=0, \\
& \frac{n^{2}}{\left(r_{h}^{2}+n^{2}\right)^{2}}\left\{\left[\left(4 g^{2} n^{2}-e^{2}\right) r_{h}+2 e g\left(n^{2}-r_{h}^{2}\right)\right] \frac{\partial}{\partial g}(\alpha+\beta)\right. \\
& \left.-2 e\left(r_{h}^{2}+n^{2}\right)(\alpha-\beta)\right\}=0, \\
& \frac{n\left(e+2 g r_{h}\right)}{\left(r_{h}^{2}+n^{2}\right)^{2}}\left[n\left(2 g n^{2}-e r_{h}\right) \frac{\partial}{\partial n}(\alpha+\beta)\right. \\
& \left.\quad-\left(e r_{h}+2 g n^{2}\right)(\alpha-\beta)\right]=0 . \\
& \frac{n^{2}}{\left(r_{h}^{2}+n^{2}\right)^{2}}\left(2 g n^{2}-e r_{h}\right)\left(e+2 g r_{h}\right) \frac{\partial}{\partial l}(\alpha+\beta)=0 .
\end{aligned}
$$

Thus, when $\alpha$ and $\beta$ satisfy the above conditions, we have completed the construction of the first law of thermodynamics. The above five expressions can be further reduced. For simplicity, we can set $x=\alpha+\beta$ and $y=\alpha-\beta$. Then, 


$$
\begin{array}{rlrl}
\frac{\partial x}{\partial r_{h}} & =\frac{\left(e^{2}+4 g^{2} n^{2}\right) y}{\left(-2 g n^{2}+e r_{h}\right)\left(2 g r_{h}+e\right)}, & & \frac{\partial x}{\partial l}=0 . \\
\frac{\partial x}{\partial e} & =\frac{2 g\left(r_{h}^{2}+n^{2}\right) y}{\left(-2 g n^{2}+e r_{h}\right)\left(2 g r_{h}+e\right)}, \\
\frac{\partial x}{\partial g} & =\frac{2 e\left(r_{h}^{2}+n^{2}\right) y}{\left(2 g n^{2}-e r_{h}\right)\left(2 g r_{h}+e\right)}, & & \frac{\partial x}{\partial n}=\frac{\left(2 g n^{2}+e r_{h}\right) y}{n\left(2 g n^{2}-e r_{h}\right)} .
\end{array}
$$

Now, based on these conditions, we can make some discussions. When $\alpha$ is equal to $\beta$, we note that $\alpha$ and $\beta$ are just constants. Especially, we find that if $\alpha=\beta=0$, it demonstrates that the asymptotic electric charge and horizon magnetic counterpart are considered as thermodynamics quantities and the general Smarr relation (61) will be reduced to Eq. (39), and similarly if $\alpha=\beta=1$, Eq. (41) are recovered. At the same time, we find that $\alpha=1, \beta=0$ or $\alpha=0, \beta=1$ cannot solve the above equations. Therefore, although the Smarr relations, with the electric and magnetic charges at infinity or on the horizon with the thermodynamic quantities, can be formulated as follows:

$$
\begin{aligned}
& M=2\left(T S-p V+\psi N^{\prime}\right)+\phi_{e} Q_{e}^{(+)}+\phi_{m} Q_{m}^{(+)}, \\
& M=2\left(T S-p V+\psi N^{\prime}\right)+\phi_{e} Q_{e}+\phi_{m} Q_{m},
\end{aligned}
$$

where $N^{\prime}=\left(N^{(1)}+N^{(2)}\right) / 2$, and the corresponding first laws cannot be established consistently.

However, for general cases where $\alpha$ and $\beta$ are functions of physical parameters $\left\{e, g, r_{h}, n\right\}$, there exist many solutions for the above equations, which correspond to different forms of thermodynamics under arbitrary choices of gauge, and here we only find some special ones. For instance, when $\beta$ is equal to some constant, we can obtain that

$$
\begin{aligned}
\frac{\partial y}{\partial r_{h}} & =\frac{\left(e^{2}+4 g^{2} n^{2}\right) y}{\left(-2 g n^{2}+e r_{h}\right)\left(2 g r_{h}+e\right)}, \\
\frac{\partial y}{\partial g} & =\frac{2 e\left(r_{h}^{2}+n^{2}\right) y}{\left(2 g n^{2}-e r_{h}\right)\left(2 g r_{h}+e\right)}, \\
\frac{\partial y}{\partial e} & =\frac{2 g\left(r_{h}^{2}+n^{2}\right) y}{\left(-2 g n^{2}+e r_{h}\right)\left(2 g r_{h}+e\right)}, \\
\frac{\partial y}{\partial n} & =\frac{\left(2 g n^{2}+e r_{h}\right) y}{n\left(2 g n^{2}-e r_{h}\right)} .
\end{aligned}
$$

It is not difficult to find that the final result is

$$
\alpha-\beta=C_{1} \frac{e r_{h}-2 g n^{2}}{n\left(e+2 g r_{h}\right)}, \quad \beta=C_{2} .
$$

Analogously, if we take $\alpha$ to be a constant, then

$$
\alpha-\beta=C_{3} \frac{n\left(e+2 g r_{h}\right)}{e r_{h}-2 g n^{2}}, \quad \alpha=C_{4} .
$$

Here, $C_{1}, C_{2}, C_{3}$, and $C_{4}$ are all arbitrary constants. Obviously, for charged Taub-NUT-AdS spacetimes containing both electric and magnetic charges, the first laws of thermodynamics and Smarr relations can be constructed in myriad forms consistently, rather than only two kinds of them as mentioned in the previous sections.

\section{CONCLUSIONS}

As shown by some recent studies [9-11], the Taub-NUT solution in the Lorentzian setup does not lead to serious problems as imagined previously, and when establishing the Smarr relation and first law of thermodynamics, the core ingredient is to introduce a pair of novel conjugate thermodynamic quantities, namely, the Misner potential and charge $\psi-N[12,16]$ to ensure that the NUT parameter $n$ varies independently. The thermodynamics of the uncharged Lorentzian Taub-NUT-AdS maintaining the present Misner strings has been formulated, and from the Komar integral notions, it turns out that $\psi$ is actually the surface gravity of the Misner strings and $N$ is interpreted as the integration over it [16]. When extended to the charged case, due to this spacetime's peculiar property as described in the previous sections, two different types of thermodynamics have been constructed by calculating the Euclidean action for free energy to determine the Misner charge and other thermodynamic quantities [20].

Inspired by the uncharged case [16] and the solution with rotation [22], in this paper we have reformulated the thermodynamic laws of the charged NUT black hole with the Misner strings present in the Einstein-Maxwell gravity from the Komar integral perspective, and shown that the Misner charges in this case are also corresponding to Komar integrals over the Misner strings. Utilizing the language of differential forms, we give the universal Smarr relation (35) directly. As a result of the distinctive property of the electric and magnetic charges illustrated in (11), the gauge freedom of the electric and magnetic potentials is nontrivial, which demonstrates that there is bound to exist a myriad of Smarr relations and meanwhile, we give the necessary conditions to satisfy the first laws consistently. Based on this philosophy, we argue that the two types of thermodynamics discussed in [20] are just the special cases of Eq. (35) under two specified gauge choices. And then, we extend this notion to more general thermodynamics when choosing other arbitrary gauges and thus obtain two novel nontrivial versions of thermodynamic laws. It is worth noting that the strategy of constructing thermodynamics of the Nutty dyonic black hole in Ref. [20] is to evaluate the Euclidean action to give the expression of free energy and then identify different thermodynamic conjugate pairs, but the determination of electric and magnetic terms is fully out of physical consideration and this technique is unconducive to generalizing to more general 
thermodynamics with the mixed horizon/infinity charges. Instead, the geometric perspective gives corresponding Komar integrals of different conjugate pairs directly and it is obvious that distinct forms of thermodynamics will be obtained only by an adjustment of the electric and magnetic potentials under certain conditions. The above results are completely different from those of the normal black holes, where the first laws of thermodynamics are not dependent on the gauge choices. However, this can be understood by the nontrivial topology of the NUT black holes. For a normal black hole, there are only two boundaries of the hypersurface connecting the horizon and asymptotic infinity, and the evaluation of the electric and magnetic charges on the horizon are the same as these at infinity. Then, the decomposition of the black hole mass will be independent on the gauge choice. While for the NUT black hole, by virtue of the existence of the Misner string, the evaluation of the electric and magnetic charges will depend on the sphere radius, which will make the decomposition rely on the gauge choice.

In summary, the geometric perspective provides us a straightforward means to establish universal thermodynamic laws for the Nutty dyonic black hole. However, many problems are still left to be further investigation, for instance, more intriguing forms of mixed charges need to be constructed, the physical meaning of Misner charge is still obscure and for the other dyonic black hole, whether we can establish different types of thermodynamics from the geometric notion. These deserve our deep explorations.

\section{ACKNOWLEDGMENTS}

This research was supported by National Natural Science Foundation of China (NSFC) with Grants No. 11375026, No. 11675015, and No. 11775022.
[1] A. H. Taub, Empty space-times admitting a three parameter group of motions, Ann. Math. 53, 472 (1951).

[2] E. Newman, L. Tamburino, and T. Unti, Empty-space generalization of the Schwarzschild metric, J. Math. Phys. (N.Y.) 4, 915 (1963).

[3] C. W. Misner, The flatter regions of Newman, Unti and Tamburino's generalized schwarzschild space, J. Math. Phys. (N.Y.) 4, 924 (1963).

[4] S. W. Hawking and G. F. R. Ellis, The Large Scale structure of Space-Time (Cambridge University Press, Cambridge, England, 1973), Vol. 1.

[5] P. Hajicek, Causality in non-Hausdorff space-times, Commun. Math. Phys. 21, 75 (1971).

[6] R. B. Mann and C. Stelea, On the thermodynamics of NUT charged spaces, Phys. Rev. D 72, 084032 (2005).

[7] C. V. Johnson, Thermodynamic volumes for AdS-TaubNUT and AdS-Taub-Bolt, Classical Quantum Gravity 31, 235003 (2014).

[8] C. V. Johnson, The extended thermodynamic phase structure of Taub-NUT and Taub-Bolt, Classical Quantum Gravity 31, 225005 (2014).

[9] G. Clément, D. Gal'tsov, and M. Guenouche, Rehabilitating space-times with NUTs, Phys. Lett. B 750, 591 (2015).

[10] G. Clément, D. Gal'tsov, and M. Guenouche, NUT wormholes, Phys. Rev. D 93, 024048 (2016).

[11] G. Clément and M. Guenouche, Motion of charged particles in a NUTty Einstein-Maxwell spacetime and causality violation, Gen. Relativ. Gravit. 50, 60 (2018).

[12] R. A. Hennigar, D. Kubiznak, and R. B. Mann, Thermodynamics of Lorentzian Taub-NUT spacetimes, Phys. Rev. D 100, 064055 (2019).

[13] J. D. Brown and C. Teitelboim, Dynamical neutralization of the cosmological constant, Phys. Lett. B 195, 177 (1987).
[14] J. D. Brown and C. Teitelboim, Neutralization of the cosmological constant by membrane creation, Nucl. Phys. B297, 787 (1988).

[15] R. Emparan, C. V. Johnson, and R. C. Myers, Surface terms as counterterms in AdS/CFT correspondence, Phys. Rev. D 60, 104001 (1999).

[16] A. B. Bordo, F. Gray, R. A. Hennigar, and D. Kubiznak, Misner gravitational charges and variable string strengths, Classical Quantum Gravity 36, 194001 (2019).

[17] D. R. Brill, Electromagnetic fields in a homogeneous, nonisotropic universe, Phys. Rev. 133, B845 (1964).

[18] J. F. Plebanski and M. Demianski, Rotating, charged, and uniformly accelerating mass in general relativity, Ann. Phys. (N.Y.) 98, 98 (1976).

[19] N. Alonso-Alberca, P. Meessen, and T. Ortin, Supersymmetry of topological Kerr-Newman-Taub-NUT-AdS spacetimes, Classical Quantum Gravity 17, 2783 (2000).

[20] A. B. Bordo, F. Gray, and D. Kubiznak, Thermodynamics and phase transitions of NUTty Dyons, J. High Energy Phys. 07 (2019) 119.

[21] A. Ashtekar and S. Das, Asymptotically Anti-de Sitter space-times: Conserved quantities, Classical Quantum Gravity 17, L17 (2000).

[22] A. B. Bordo, F. Gray, R. A. Hennigar, and D. Kubiznak, The first law for rotating NUTs, Phys. Lett. B 798, 134972 (2019).

[23] D. Kastor, S. Ray, and J. Traschen, Enthalpy and the mechanics of AdS black holes, Classical Quantum Gravity 26, 195011 (2009).

[24] D. Kubiznak and P. Krtous, On conformal Killing-Yano tensors for Plebanski-Demianski family of solutions, Phys. Rev. D 76, 084036 (2007).

[25] V. Frolov, P. Krtous, and D. Kubiznak, Black holes, hidden symmetries, and complete integrability, Living Rev. Relativity 20, 6 (2017). 